\title{
Médiévales
}

Langues, Textes, Histoire

70 | printemps 2016

Lieux d'hygiène et lieux d'aisance en terre d'Islam (VII $\mathrm{e}_{-}$ $\mathrm{XV}^{\mathrm{e}}$ siècle)

\section{Usages de bienséance et règles de pureté dans la littérature juridique de l'Occident musulman médiéval}

Rules of Polite Society and Norms of Purity in the Jurisprudential Literature of Medieval Muslim West

\section{Yassir Benhima}

\section{OpenEdition}

\section{Journals}

Édition électronique

URL : http://journals.openedition.org/medievales/7696

DOI : 10.4000/medievales.7696

ISSN : 1777-5892

\section{Éditeur}

Presses universitaires de Vincennes

\section{Édition imprimée}

Date de publication : 23 juin 2016

Pagination : 13-23

ISSN : 0751-2708

\section{Référence électronique}

Yassir Benhima, «Usages de bienséance et règles de pureté dans la littérature juridique de l'Occident musulman médiéval », Médiévales [En ligne], 70 | printemps 2016, mis en ligne le 15 juin 2018, consulté le 03 mai 2019. URL : http://journals.openedition.org/medievales/7696 ; DOI : 10.4000/ medievales.7696 
Yassir Benhima

\section{Usages de bienséance et règles de pureté dans la littérature juridique de l'Occident musulman médiéval}

L'étude des systèmes de pureté et des règles de purification en islam demeure un sujet peu abordé par les historiens, qui ont longtemps délaissé ce terrain considéré comme relevant du seul domaine de l'anthropologie des religions ou encore de l'histoire du droit. Les raisons d'un tel désintérêt se trouvent notamment dans une vision dominante dans l'ethnologie du $\mathrm{XIX}^{\mathrm{e}}$ siècle, envisageant - à l'image de William Robertson Smith - les rituels de purification comme une «survivance » de pratiques magiques ou superstitieuses liées à des croyances anciennes ${ }^{1}$. Le renouvellement de l'approche de la question de la pureté suscité par les travaux de Mary Douglas $^{2}$ n'a pas immédiatement concerné les spécialistes de l'islam³ ${ }^{3}$. Mais depuis deux décennies, de nombreux travaux, essentiellement anglosaxons, explorent plusieurs questions relevant des systèmes de pureté dans ce contexte particulier, permettant ainsi de sortir le thème du rituel d'une intemporalité dans laquelle il a été relégué, aussi bien par la pensée religieuse traditionnelle que par l'ethnologie.

En effet, dans l'islam comme dans de nombreuses traditions religieuses d'origine orientale, les catégories du pur et de l'impur conditionnent le rapport de l'homme à son corps et à la nature en général, et

1. Sur l'attitude des premiers ethnologues des religions à l'égard des systèmes de pureté : Z. MAGHEN, « Much Ado about Wuḍū’ », Der Islam, 76 (1999), p. 205-252 (p. 206209).

2. Notamment par son ouvrage Purity and Danger, Londres, 1966 (traduction française : M. Douglas, De la souillure. Essai sur les notions de pollution et de tabou, Paris, 1971).

3. Le premier à avoir attiré l'attention sur une nécessaire relecture du système de pureté en islam, notant l'inadéquation en la matière des thèses de M. Douglas, est A. KEVIN REINHART, «Impurity/No Danger », History of Religions, 30/1 (1990), p. 1-24. 
déterminent un nombre de prescriptions et d'interdits ${ }^{4}$. Trois domaines parallèles relèvent de cette classification : l'alimentation ${ }^{5}$, la sexualité et les excrétions corporelles. Ces deux dernières catégories entraînent des situations d'impureté, exigeant du croyant l'accomplissement d'un rituel de purification plus ou moins codifié. Les relations sexuelles (ainsi que les excrétions corporelles liées à la fonction sexuelle ou procréative) sont à l'origine d'une souillure majeure ( $\breve{g} a n a \bar{a} b a)$, nécessitant, pour se purifier, le recours au gissl, lotion du corps entier. Quant au hadat, il englobe toute substance (liquide, solide ou gazeuse) sortant de l'anus, de l'urètre ou du vagin, et empêchant l'accomplissement de la prière canonique (salät $)^{6}$. Cette souillure mineure peut être éliminée grâce au $w u d \bar{u}^{\prime}$ ' (ablution), dont l'origine pourrait remonter à une tradition zoroastrienne ${ }^{7}$. Parmi les causes de l'impureté mineure, les excréments occupent une place particulière, dont témoignent de nombreuses mentions, principalement dans les sources juridico-religieuses, permettant de discerner le caractère complexe du système de pureté musulman.

Si l'arrivée du hadat engendre un état d'impureté rituelle, la limitation des effets de cet état et l'élimination de tout résidu d'impureté touchant le corps après l'expulsion des urines ou des excréments impliquent une série de règles qui définissent un véritable code de bienséance. D'un point de vue juridique, il ne faut pas confondre les ablutions, le $w u d \bar{u}^{\prime}$, rite obligatoire $(\operatorname{fard})^{8}$, avec d'autres actes de purification qui ne constituent

4. Une approche comparative des systèmes de pureté rituelle dans les trois grands monothéismes est proposée dans : «Les règles de pureté », dans D. ALBERA et K. BERTHELOT (éd.), Dieu, une enquête. Judaïsme, christianisme, islam, ce qui les distingue, ce qui les rapproche, Paris, 2013, p. 557-636.

5. M. H. BENKHEIRA, Islâm et interdits alimentaires. Juguler l'animalité, Paris, 2000.

6. D. Albera et K. Berthelot (éd.), Dieu, une enquête..., p. 584 ; E. Chaumont, « Pureté rituelle », dans M.-A. AMIR MoEzzI (éd.), Dictionnaire du Coran, Paris, 2007, p. 713-715.

7. M. H. KATZ, « Cleanliness and Ablution », Encyclopaedia of the Qur'ān, Leyde, 2001, 1, p. 341-344.

8. Le caractère obligatoire du $w u d \bar{u}^{\prime}$, préalablement aux actes de dévotion, est partagé par les différentes doctrines musulmanes. Plusieurs divergences demeurent en revanche sur les détails de son accomplissement. Certaines écoles considèrent par exemple que le rinçage de la bouche (madmada) et le lavage du nez en aspirant puis rejetant l'eau par les narines (istinšă $q$ et istinfār) ne sont pas des actes obligatoires. La purification des pieds, obligatoire par ailleurs, peut se faire par lavage chez les Sunnites ou par madéfaction (friction humide : mash) chez les Chiites. Sur les différentes interprétations et nuances à propos des gestes du wudü', on peut lire avec un grand intérêt la synthèse proposée par IBN RUŠD, Bidāyat al-muğtahid wa nihāyat al-muqtașid, Le Caire, 1995, t. I, p. 25-97. Sur le rituel des ablutions en général, on lira Z. MAGHEN, «Ablution », Encyclopaedia of Islam, vol. 3 (consulté sur Brill Online le 21 mars 2015) ; pour le rite mālikite plus spécifiquement, voir G. H. BOUSQUET, «La pureté rituelle en islam (étude de figh et de sociologie religieuse) », Revue de l'histoire des religions, 138/1 (1950), p. 53-71. Les divergences des exégèses sunnite et chiite des versets coraniques portant sur le $w u d \bar{u}$ ', et en particulier sur la purification des pieds, sont 
qu'une coutume recommandée, sunna, puisant sa source d'exemplarité dans les comportements de Muhammad et de ses compagnons. D'ailleurs, les adversaires de Muhammad moquaient cette attitude, notamment dans un récit (habar) récurrent dans lequel ils demandaient à Salmān al-Fāris̄̄, l'un des plus proches compagnons du prophète, si ce dernier enseignait à ses partisans l'art et la manière de déféquer ( hirā'a). La réponse affirmative de Salmān permet aux juristes des époques ultérieures de faire remonter l'origine des règles de bienséance, progressivement constituées, aux temps de Muhammad, voire au temps originel de l'inné, la fitra ${ }^{9}$.

Ainsi, les ouvrages de droit ${ }^{10}$, qui commencent généralement par le kitāb al-țahāra (livre ou chapitre de la pureté rituelle), consacrent une littérature relativement importante aux règles de bienséance (adab qadă ' $a l-h \bar{a} \breve{g} a$ ) et à l'élimination des restes d'urine et d'excrément (istiğmār et istinğ $\left.\bar{a}^{\prime}\right)$. C'est cette littérature qui fournira ici notre principale source documentaire, puisée principalement (mais pas exclusivement) dans des ouvrages de droit malikite, l'école dominante en Occident musulman. La sensibilité des juristes au respect de la pureté s'exprimait par un ensemble de mesures encadrant ces pratiques qui touchent au choix des lieux, des postures et de la gestuelle.

\section{Les latrines de Muhammad}

Quand on examine la littérature juridique, on est frappé par l'aspect très vague des appellations concernant les lieux d'aisance. Les rapports remontant aux premières autorités religieuses de l'islam, à l'époque de Muhammad, de ses compagnons et des premières générations de juristes, insistent surtout sur le choix d'un endroit préservant l'intimité (sātir) et éloigné $^{11}$; on déconseille généralement les lieux publics, au bord de la

étudiées par J. BURTON, « The Qur'ān and the Islamic Practice of Wudūu' », Bulletin of the School of Oriental and African Studies (BSOAS), 51/1 (1988), p. 21-58. Pour un examen détaillé de la fabrique de la norme en matière de purification rituelle dans le droit sunnite, voir M. H. KATZ, Body of Text. The Emergence of the Sunnī Law of Ritual Purity, New York, 2002.

9. La notion de fitra (nature innée) décrit l'état originel d'un être humain avant sa corruption par son environnement et suppose l'existence d'une « conception religieuse originelle » : voir G. GOBILLOT, « Nature innée », dans M.-A. AMIR MOEZZI (éd.), Dictionnaire du Coran, Paris, 2007, p. 591-595.

10. Le mot «droit » est utilisé ici pour rendre le terme arabe fiqh. Les ouvrages exploités relèvent généralement de la riche littérature des $f u r \bar{u}^{`}$ (droit appliqué) qui a connu un grand développement chez les Mālikites en Occident musulman médiéval. Ces ouvrages de furū comportent plusieurs types : les recueils de consultations juridiques $($ fat $\bar{a} w \bar{a})$; les manuels de hisba ; et les traités de casuistique, détaillés (muṭawwalāt) ou abrégés (muhtașarāt).

11. IBN ŠĀS, 'Iqd al-ğawāhir al-tamīna fi madhab 'ālim al-madīna, Beyrouth, 1995, I, p. 47. 
route, à l'ombre d'un arbre ou contre les murs, ou encore près des cours d'eau. Ainsi, il fallait éviter de faire ses besoins sur les rives de fleuves et surtout près de l'eau « stagnante ${ }^{12} »$.

En l'absence de mentions claires de latrines dans le contexte de l'islam primitif, les récits renvoient surtout à un milieu peu urbanisé où uriner et déféquer en rase campagne (falāt) étaient une habitude courante, sinon la norme. Cette situation explique pourquoi la terminologie de la défécation en arabe emprunte nombre de ses mots au champ sémantique

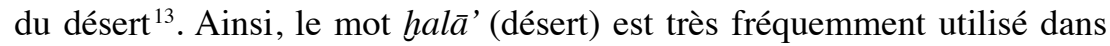
les traditions attribuées à Muḥammad et à ses compagnons pour qualifier le lieu où l'on fait ses besoins, tandis que l'acte lui-même est désigné par le mot tahallī. Cet usage se perpétuera par la suite dans l'appellation «bayt al-halā' », qui désigne une latrine.

Ce constat se confirme à la lecture du principal verset coranique instituant l'obligation d'abluer après la défécation. En effet, l'expression coranique « í $\underline{d} \bar{a} \breve{g} \bar{a}$ 'a ahadun min al- $\dot{g} \bar{a}$ 'it $»$ renvoie à l'idée de fréquenter un espace garantissant l'intimité afin de déféquer ${ }^{14}$. Le terme $\dot{g} \bar{a}$ 'it désigne littéralement un terrain en dépression ; ce n'est que par métonymie que le nom du lieu sera également étendu aux excréments. Un autre terme, kanīf, assez fréquent dans les textes de l'Occident musulman pour désigner un puits perdu ou une latrine, qualifiait dans l'arabe des Bédouins un enclos, rendant généralement l'idée de couvrir, garder ou protéger l'intimité.

Ce que suggère le champ sémantique des plus anciens termes relatifs à la défécation en dehors de latrines, donc en l'absence d'un espace construit réservé et éventuellement doté d'eau, est confirmé par des témoignages concernant Muhammad, 'Umar ou 'Alī ${ }^{15}$. Notons enfin que le mot mirhạa (« latrine ») est absent dans les traditions attribuées à Muhammad et ses compagnons en lien avec le contexte arabique. On le trouve pourtant dans un récit relatant le témoignage d'al-Anșārī, un compagnon de Muhammad, qui a constaté que les latrines (marāḥ̄ $)$ ) en Syrie (probablement du temps

12. Ibid.

13. Pour l'enquête lexicographique, le Lisān al-'arab d'Ibn Manẓūr est le principal dictionnaire utilisé ici. Il est aujourd'hui accessible en ligne (par exemple sur www.baheth. info).

14. Verset 6 de la sourate V (« la Table » : al-mā'ida). La traduction de B. KAZIMIRSKI, Le Coran, Paris, 1970, p. 106 (« quand vous aurez satisfait vos besoins naturels »), ne rend pas l'ambivalence de l'expression arabe ; J. BERQUE (Le Coran. Essai de traduction, Paris, 2002 [1 ${ }^{\text {re }}$ éd. 1990], p. 122), le traduit par : «(si) vous revenez de la selle », choix qui semble plus adéquat.

15. Plusieurs anecdotes sont citées par nos sources à ce propos. Par exemple, Muhammad aurait été vu par son beau-frère, 'Abd Allāh b. 'Umar, dans la maison de Hafṣa, l'une des épouses du prophète, alors qu'il faisait ses besoins en se tenant sur (ou entre) deux briques crues (labina) : IBN ABĪ ZAYD AL-QAYRAWĀNī, Kitāb al-nawādir wa al-ziyādāt, Beyrouth, 1999, 1, p. 22. 
des conquêtes) étaient souvent orientées vers la qibla. On peut supposer que ces latrines étaient équipées de canalisation pour l'évacuation des eaux usées, car la racine rḥ d renvoie à l'idée de laver avec de l'eau.

La question de l'orientation en faisant ses besoins a suscité aussi beaucoup de commentaires. Sans rentrer dans les détails des récits, on peut en conclure qu'il était recommandé d'éviter de se diriger vers la qibla, même si certains rapports évoquent que Muhammad lui-même était peu précautionneux à cet égard quand il s'agissait de Jérusalem, première qibla des musulmans. Selon Mālik (m. 796), cette recommandation s'appliquait seulement dans le cas d'uriner ou déféquer en rase campagne (falät) et sur les terrasses ; en revanche pour les latrines construites (marāhī $\bar{d}$ ), l'orientation n'importe pas ${ }^{16}$.

Les sources juridiques fourmillent également de récits concernant les relations entre les lieux de défécation et le monde des ğinn. Il faut noter à cet égard la proximité linguistique entre les deux faits à travers la racine $h b \underline{t}$ : si le mot habīt est utilisé, par opposition à tayyib, pour désigner les aliments impurs et toute forme de souillure, il exprime également l'idée de la présence de ğinn et d'êtres malveillants. Cette corrélation explique pourquoi des formules protectrices devaient être prononcées avant d'aller faire ses besoins, usage qui se perpétue dans les pratiques relatives aux latrines plusieurs siècles après l'arrivée de l'islam. Ces traditions pouvaient également être étendues à la recommandation de s'abstenir de parler, de réciter un poème ou même de lire en se soulageant ${ }^{17}$.

\section{L'istiğmār et l'istinğāa'}

Outre le lieu et ses implications, les textes détaillent également la manière de se nettoyer après avoir fait ses besoins et avant l'accomplissement du wuḍu'. Il s'agit de deux procédés : l' 'istiğmār et l'istinğ $\bar{a}$ '.

L'istiğmāar, de ğimār («pierres »), désigne l'utilisation de pierres ou d'autres objets solides pour éliminer ou essuyer les restes d'excréments après la défécation. Les sources juridiques rapportent à cet effet les recommandations de Muhammad d'utiliser au minimum trois pierres afin de garantir l'élimination de la souillure ; le nombre de pierres peut être plus important, mais doit toujours être impair (pour des raisons non précisées par les sources, mais relevant vraisemblablement de superstitions). Les premières autorités malikites, citées par Ibn AbīZayd al-Qayrawān̄i (m.996), divergent sur la nature des objets utilisés. Si Mālik tolère l'utilisation d'un os ('Umar par exemple avait son os dans l'endroit où il avait ses habitudes),

16. Ibid., p. 21.

17. Pour une présentation détaillée, voir IBN ŠĀs, 'Iqd al-ğawāhir al-țamīna..., p. 47-48. 
du charbon de bois, voire l'excrément d'un animal pour l'isti ğmār , d'autres juristes attribuent à Muḥammad l'interdiction d'employer le bois, le charbon de bois, le cuir, le tissu ou encore les déjections animales pour ôter la souillure. Les ossements, considérés comme des déchets alimentaires, sont également frappés d'interdits ${ }^{18}$. Selon plusieurs témoignages remontant à l'époque de Muhammad, l'on pouvait effectuer l'istiğmār à la manière de 'Umar ibn al-Hattāab, en s'essuyant contre un mur'19.

Cette pratique de l'istiğmār, clairement associée à un milieu marqué par la pénurie d'eau, est incontestablement liée au mode de vie bédouin. L'expansion de l'islam limitera son utilisation, car les juristes s'accordent sur la préférence de la deuxième méthode, l'istinğ $\bar{a}$ ', qui utilise l'eau. D'ailleurs, dès l'époque d'Ibn Šihāb al-Zuhrī (m. 723), on décrit l'istiğmār comme une pratique ancienne et délaissée ${ }^{20}$. Cela signifie que l'isting $\breve{a} \bar{a}$, nécessitant l'utilisation de l'eau pour éliminer les restes d'excrément ou d'urine, s'est imposé comme pratique courante. Les juristes malikites, dans plusieurs des ouvrages classiques de l'école, s'étendent sur les différents procédés recommandés pour effectuer la lotion par l'eau des parties intimes après l'urine et la défécation ${ }^{21}$.

\section{Une lecture ésotérique de la purification}

Les sources juridiques malikites abordent d'une manière détaillée ces formes de toilette et toute la casuistique de la souillure par les excréments humains et animaux (en marchant dans les rues ; éclaboussures sur les vêtements en faisant ses besoins ; souillure de l'eau au contact des excréments d'animaux, etc.). Tout cela révèle une obsession de la pureté dont l'expression demeure très technique et peu portée sur la théorie ou sur l'interprétation ésotérique. Il n'est pas étonnant, d'ailleurs, de ne pas retrouver chez ces auteurs des indications sur les aspects symboliques de la țahāra. Heureusement, un autre auteur maghrébin, Abū Ḥan̄ifa al-Nu'mān

18. Des débats sur le statut des aliments cuits par le feu et sur l'éventualité d'une impureté découlant de leur consommation ont divisé les autorités juridiques de l'islam des origines : voir M. H. KATZ, Body of Text..., p. 101-123.

19. 'ALĀ' AL-Dīn, AL-Hindī (m. 1567), Kanz al-'ummāl fì sunan al-aqwāl wa al-af'āl, Hyderabad, 1960, 9, p. 519.

20. D'après IBN ABĪ ZAYD AL-QAYRAWĀNī, Kitāb al-nawādir..., 1, p. 23-25, al-Zuhrī aurait déclaré : «șāra 'amalu al-nās hilāfahu».

21. À l'image d'Ibn Abī Zayd al-Qayrawān̄̄, qui apporte quelques détails « techniques » sur la gestuelle à adopter pour ôter la souillure (Kitāb al-nawādir, 1, p. 26-27). L'auteur insiste néanmoins sur le fait que l'istinğ $\bar{a}$ ' ne constitue par un devoir (farḍ), mais qu'il relève tout simplement de la nécessité de se laver, préalablement à la purification rituelle. Il n'implique pas par ailleurs l'intention (niyya) du fidèle, nécessaire pour l'accomplissement de tout acte de dévotion. 
(m. 974), grand cadi des Fatimides et fondateur du droit isma'ilite ${ }^{22}$, nous livre une interprétation inédite des rites déjà présentés. Rappelons que, pour les Isma'ilites, toute réalité, y compris juridique, revêt deux niveaux : le $z \bar{a} h i r$ apparent ou obvie, qui coïncide avec le niveau littéral des textes, et le bāṭin, sens caché accessible uniquement aux détenteurs du savoir ésotérique (les imām-s, seuls aptes à le transmettre à leurs proches). Ainsi, al-Nu'mān nous livre sur la țhāra deux versions complémentaires : l'une, contenue dans Da 'á'im al-islām (piliers de l'islam), est classique et assez conforme à ce que 1 'on trouve dans les sources sunnites ${ }^{23}$. La seconde version, censée refléter le bātin, est présentée dans son $T a$ 'wīl da 'à'im al-islām (interprétation des piliers de l'islam) ${ }^{24}$.

Ainsi, pour le fondateur du droit isma 'ilite, la conduite recommandée pour faire ses besoins naturels a un sens profond. D’abord, les excrétions corporelles entraînant la souillure du fidèle sont assimilées à des formes d'incroyance. Si l'urine symbolise l'associationnisme (širk: terme signifiant le fait d'associer d'autres divinités à la vénération d'Allāh), les excréments renvoient à l'infidélité (kufr), alors que les flatulences équivaudraient à l'hypocrisie (nifāq) et à l'incertitude. La foi ( $\bar{\imath} m \bar{a} n)$, représentée par l'état de pureté, n'est accessible que grâce à la purification par la science ('ilm), qui incarne d'un point de vue ésotérique l'eau utilisée dans le rituel canonique.

Cette purification indispensable a lieu dans les latrines (bayt al-halä'), lieu où l'on se débarrasse de la souillure, et correspond dans le bātin à la $d a$ 'wa, prédication permettant de rejeter l'incrédulité pour embrasser la foi. Fréquenter des latrines, comme suivre l'initiation à la doctrine isma'ilite, implique le respect d'un certain nombre de règles dont la connaissance est indispensable pour la complétude de la foi. Aussi est-il recommandé d'entrer dans les latrines avec des chaussures, geste interprété comme synonyme de la maîtrise du zăhir. En effet, seul un musulman connaissant le sens exotérique de la Loi peut être initié par la $d a^{\prime} w a$ isma'ilite, qui lui ouvre la voie du sens caché. En entrant dans les latrines du pied gauche, et en s'appuyant sur ce même pied lorsqu'il défèque, le fidèle se départit de la souillure (et donc de l'infidélité qui l'incarne) en s'appuyant sur l'enseignement d'un hŭğğa, prédicateur qui agit comme la preuve de la véracité de la foi. C'est de la main gauche, aussi, qu'il faut accomplir les différents gestes de purification, rappelle le qāḍ̄ al-Nu'mān, notamment l'isting $\bar{a}$ '. L'eau purificatrice utilisée ne doit pas être versée directement sur les excréments dans le canal d'évacuation, de manière à éviter de mélanger

22. La personnalité et le rôle du Qāḍī al-Nu'mān, par ailleurs auteur prolifique, nous sont bien connus : voir par exemple S. Hamdani, Between Revolution and State. The Path to Fatimid Statehood, Londres, 2006, et G. Cilardo, The Early History of Ismaili Jurisprudence. Law Under the Fatimids, Londres, 2012.

23. AL-QĀDì AL-NU'MĀN, Da 'â' 'im al-islām, Beyrouth, 1995, 1, p. 145-147.

24. AL-QĀDī AL-NU'MĀN, Ta'wìl da 'à'im al-islām, Beyrouth, 2006, 1, p. 39-57. 
la science et l'infidélité. En s'abstenant de parler dans les latrines, acte destiné en apparence à éviter de prononcer des mots liés au sacré dans un lieu de souillure, le fidèle se met, selon le juriste isma'ilite, à l'écoute du $d \bar{a}^{`} \bar{l}$, prédicateur chargé de son initiation ésotérique.

Ce texte isma'ilite, qui s'illustre par sa lecture symbolique très éloignée de la facture technique de la littérature juridique dominante (chez les sunnites notamment), montre toute la portée conceptuelle et intellectuelle que peut incarner le rituel de la purification corporelle. Cette importance donnée à la notion de pureté trouve un écho particulier dans le chiisme, où la personne de l'imām est considérée comme un être pur (țăhir).

\section{La souillure dans la ville : normes, pratiques et représentations}

Si les textes juridiques expriment une attention particulière aux respects des normes de la tahāra, cet idéal de pureté est bien perceptible également dans les représentations du fait urbain. En effet, par la concentration d'habitants qu'elle implique, la ville est aussi confrontée aux problèmes de gestion des déchets humains, et en particulier des excréments. La gestion de la souillure se trouve ainsi conditionnée non seulement par le contenu de la norme, mais aussi par des contraintes pratiques et des représentations collectives.

Même si les sources sont peu disertes à ce sujet, il semble que tout métier impliquant la manipulation des excréments est taxé de vil, à l'instar de la profession de vidangeur, indispensable mais ingrate. À Siğilmāsa, à en croire l'anonyme de l'Istibṣār (écrit à la fin du XII ${ }^{\mathrm{e}}$ siècle), le kannāf (« vidangeur ») exerce un métier vil ( $r a d \underline{\imath} l)$, autant que la maçonnerie, qui est réservé aux juifs de la ville ${ }^{25}$.

Au-delà du métier lui-même, la crainte de la souillure provoquée par la vidange des lieux d'aisance concerne la salubrité de la rue, mais aussi le confort de ses usagers, comme en témoignent les dispositions préconisées par les juristes. Pour Malaga, al-Saqați exige que les transporteurs du contenu des puits perdus (kunaf) soient tenus de couvrir leurs seaux ( $a k w \bar{a} b$, pl. de $k \bar{u} b$, qui semble désigner un seau en bois) ; chaque $k \bar{u} b$ doit être porté par deux personnes pour qu'il ne puisse pas toucher les passants ; on doit également sonner une cloche pour alerter les passants de la nécessité de s'écarter ; de même, il est interdit que l'un des porteurs puisse transporter au même moment deux seaux en raison du préjudice qu'il pourrait faire subir

25. ANONYME, Al-Istibșār fì 'ă̆ā'ib al-amșār, Casablanca, 1985, p. 202. Sur les métiers vils en Islam, voir R. BRUNSCHVIG, « Métiers vils en Islam », Studia Islamica 16 (1962), p. 41-60. 
aux passants ${ }^{26}$. Plus tôt, Ibn 'Abdūn avait également préconisé l'utilisation des $a k w a \bar{a} b$, même si son texte semble plutôt indiquer qu'à Séville l'usage était d'employer des paniers ; l'auteur sévillan exige ainsi que ces paniers soient imperméables, car d'éventuelles infiltrations pouvaient incommoder les passants ${ }^{27}$.

S'ils constituent un élément inspirant dégoût et méfiance, les excréments peuvent se transformer en un ingrédient utile, voire rentable. Les données sont certes sporadiques, mais montrent au moins, dans certains cas, la réutilisation possible du contenu des vidanges. Le cas de Tozeur, dans le sud de l'Ifrīqiya, est emblématique à ce propos. Les gens de Tozeur vendent le zabal (terme généralement utilisé pour désigner le fumier) provenant de leurs latrines (marăhî̀ ) ; on les injurie pour cela car ils n'utilisent pas d'eau dans les latrines de peur que le fumier ne soit altéré. Ainsi, après être allé au mirhạa , l'on se rend à une rigole pour se laver. L'acheteur du fumier fait le tour des maisons avec un récipient et choisit les excréments secs, qui rapportent bien plus que ceux touchés par l'eau. L'aspect lucratif de la chose a poussé les habitants à aménager des latrines dans leurs jardins, lorsqu'ils jouxtent les voies fréquentées, afin que les étrangers ou toute personne ayant un besoin pressant puissent se soulager. Mais les gens du pays ont l'habitude de se retenir un jour ou deux, réservant leurs selles à leurs propres latrines. Tout cela s'explique par la sécheresse de leurs terres proches du désert ${ }^{28}$.

À Séville, Ibn 'Abdūn évoque clairement la destination du contenu des puits perdus : ce dernier devait être acheminé à l'extérieur des portes des villes, soit dans les champs et les jardins (comme fertilisant) ou dans d'autres lieux réservés à cet usage ${ }^{29}$. La pratique était connue aussi à Tudèle, où al-Tuṭaylī, au cours du $\mathrm{X}^{\mathrm{e}}$ siècle, traite de la question de la propriété commune du contenu d'une fosse d'aisance, dont la vente nécessite l'accord des deux voisins ${ }^{30}$. L'utilisation des excréments humains comme combustible n'est pas attestée ${ }^{31}$. Par ailleurs, le recueil de fatāwā d'al-Burzulī (1438)

26. Al-SAQAṬī, Kitāb fì ādāb al-hisba, éd. G.-S. Colin et É. LÉVI-PROVENÇAL, Un manuel hispanique de hisba, Paris, 1931, p. 67 du texte arabe. Sur le sens du mot küb, p. 61 du glossaire.

27. IbN 'ABdūn, Risāla fì al-hishba, dans : É. LÉvi-ProvençAL (éd.), Talāt rasā'il andalusiyya fì al-hisba, Le Caire, 1955, p. 38.

28. ANONYME, Al-Istibșār..., p. 156.

29. IBN “ABDŪN, Risāla fì al-hisba, p. 37.

30. AL-TUṬYYLİ, Al-Qad̄ā’ bi-l-marfiq fì al-mabān̄̄ wa nafy al-ḍarar, Rabat, 1999, p. 104-106.

31. Plusieurs textes évoquent néanmoins l'utilisation des excréments d'animaux à cet usage, notamment pour la cuisson des aliments. Si les soufis y voyaient généralement un acte de dépouillement, cette utilisation pouvait susciter les réserves des juristes craignant la souillure des aliments au contact du combustible. Voir par exemple, IBN ABĪ ZAYD AL-QAYRAWĀNĪ, Kitāb al-nawādir..., 1, p. 83. 
évoque leur emploi comme enduit imperméabilisant. Les cendres des excréments peuvent ainsi entrer dans la composition de matériaux utilisés pour assurer l'étanchéité des toitures, ou dans la composition d'un mortier hydrofuge employé dans les ouvrages hydrauliques. Dans les deux cas, le juriste tunisois conclut à la licéité de la pratique, tolérant un degré selon lui négligeable de souillure ${ }^{32}$.

Si la régulation de la présence des excréments (et de toute forme de souillure) dans l'espace urbain est synonyme d'une bonne gestion de la ville, une gestion respectant l'idéal de la tahāra et luttant contre toute nuisance (darar), les représentations du désordre urbain se réfèrent parfois aux excréments. Observons comment, par exemple, Ibn al-Hațî̉ (1313-1374), vizir et grand polygraphe grenadin, dénigre dans deux vers de poésie la ville de Meknès qu'il visita lors de son exil marocain au début des années 1360 :

Les remparts de Meknès sont rapiécés / à l'instar des vêtements de ses habitants

Des maisons en ruine sur des mers d'excréments / son état convient à ceux qui l'habitent ${ }^{33}$

Le délabrement de la muraille de Meknès et la saleté de ses rues, constatés par l'auteur dans la Nufādat al-ğirāb fì 'ulālat al-igitirāb, font écho à la description que Ibn al-Hațīb fait de cette même ville dans une autre œuvre, où il remarque que sa boue collante macule les vêtements des gentilshommes (z̧urafā') tellement la saleté (qadar) imprègne ses rues ${ }^{34}$.

À travers les différents éléments exposés ici, on aperçoit une évolution vers une codification de plus en plus forte des règles de défécation et de toilette préalable au $w u d \bar{u}^{\prime}$. Ne relevant pas d'une obligation canonique, ces règles constituent l'expression d'une étiquette (adab) trahissant les mutations sociétales des premiers musulmans. Les pratiques issues du contexte bédouin impliquaient souvent l'absence de latrines construites et la défécation à l'air libre là où un minimum d'intimité était accessible. Par ailleurs, la rareté de l'eau faisait de la pratique de l'isti ğmār une solution efficace utilisant ce qui est le plus facilement accessible dans un milieu désertique, des pierres. Ce recours à des éléments solides dans un rituel de purification se maintient par ailleurs dans les ablutions sèches, tayammum, considérées comme une alternative légale en cas d'absence d'eau.

32. Al-Burzulī, Ğāmi ‘ masā’il al-ahkām, Beyrouth, 2002, 1, p. 192-93. Le juriste utilise deux termes différents pour qualifier ces cendres : le națūn, pour les toitures, et $a l-s ̌ a h b \bar{a}$, , pour la composition du mortier hydraulique, istāa .

33. IBN AL-HुAṬ̄̄B, Nufādat al-ğirāb fì 'ulālat al-ig̀tirāb, 3, Casablanca, 1989, p. 95-96.

34. IBN AL-HATṬ̄B, $M i$ 'yār al-ihtiyār fì dikr al-ma ‘āhid wa al-diyār, Rabat, 1977, p. 78. 
L'expansion de l'islam au-delà de son foyer arabique marquera le début d'un processus de complexification des pratiques et rites de purification. D'abord, les latrines s'imposent comme des lieux garantissant l'intimité et facilitant la toilette, avec une préférence manifeste pour l'isting $\breve{a}$ ' utilisant exclusivement de l'eau ${ }^{35}$. Elles deviennent un marqueur urbain par excellence, permettant de réguler les conditions d'évacuation des matières fécales dans l'espace. Quand la ville ne peut assurer cette fonction vitale, les excréments envahissent l'espace et entraînent un désordre urbain. Ce processus, qui n'est pas sans rappeler les mutations de la société occidentale en la matière, comme les décrivait Norbert Elias dans son ouvrage sur le «processus de civilisation », est le résultat d'un travail de construction progressive de la norme, mais aussi des représentations qui la sous-tendent ${ }^{36}$. Il témoigne par ailleurs de la nécessité d'une historicisation de l'approche du rituel musulman, longtemps abordé comme une réalité intemporelle.

\section{Yassir Benhima - Université de Paris III-Sorbonne Nouvelle/UMR 5648-CIHAM}

\section{Usages de bienséance et règles de pureté dans la littérature juridique de I'Occident musulman médiéval}

Cet article étudie les règles de bienséance, leur codification et leur signification dans la tradition juridique de l'Occident musulman médiéval. Préconisées préalablement au rituel canonique des ablutions, ces normes explicitent le comportement recommandé, notamment lors de la fréquentation des latrines. N'ayant pas un caractère obligatoire, ces règles s'imposent néanmoins parallèlement à la naissance d'une société urbanisée et répondent au souci permanent de la pureté qui préoccupe les juristes.

Ablutions - excréments - islam - pureté/impureté - purification rituelle

\section{Rules of Polite Society and Norms of Purity in the Jurisprudential Literature of Medieval Muslim West}

This article studies the rules of good behaviour concerning purity, their codification and meanings in the juristic literature of Medieval Muslim West. Recommended before the canonical ritual of ablutions, these norms describe the ideal behaviour to follow, in particular in latrines. Not obligatory, these rules were although largely diffused in parallel to the emergence of an urbanized society and also as response to the permanent preoccupation of jurists about purity.

\section{Ablution - Excrements - Islam - Purity/Impurity - Ritual of purification}

35. Une évolution similaire peut être constatée à propos du hammām, qui suscita d'abord les réserves des juristes avant d'être pleinement adoptée dans les usages des musulmans : voir M. H. BENKHEIRA, « Hammām, nudité et ordre moral dans l'islam médiéval » Revue de l'histoire des religions, 3/2007 (2007), p. 319-371, et 1/2008 (2008), p. 75-128.

36. N. Elias, La Civilisation des mours, Paris, 2003. Le titre original de l'ouvrage est : Über den Prozess der Zivilisation. 
